\title{
Experiencia Emocional Subjetiva en Deportistas Extremos: Estudio Cualitativo
}

\section{Emotional Subjective Experience in Extreme Sports Participants: A Qualitative Study}

\section{Experiência Emocional Subjetiva em Atletas Extremos: Um Estudo Qualitativo}

\author{
Cajina - Guedeat, M. ${ }^{1}$; Reyes - Bossio, M. ${ }^{2}$ \\ ${ }^{1,2}$ Universidad Peruana de Ciencias Aplicadas, Facultad de Psicología, Lima, Perú
}

\section{RESUMEN}

Los participantes en deportes extremos usualmente han sido estudiados desde la perspectiva de riesgo. El presente estudio busca alejarse de dicha preconcepción y tiene como objetivo estudiar la experiencia subjetiva emocional. Se empleó una metodología cualitativa con diseño fenomenológico-hermenéutico tomando como herramienta un sistema de categorías construido a partir de los sistemas conversacionales. Los informantes fueron 8 deportistas extremos pertenecientes a un mismo grupo. Dentro de los deportes extremos se incluyen: escalada de montaña, rapel, puenting, montañismo y bungee jumping. Los resultados indican que el miedo es generador de libertad, puede ser útil, es gratificante y también es promotor de transformaciones personales. Esta investigación otorga valor teórico al porqué del involucramiento en deportes extremos.

Palabras clave: deporte extremo, experiencia subjetiva, miedo, transformación

\begin{abstract}
Participants in extreme sports have usually been studied from a risk perspective. The present study seeks to move away from this preconception and aims to study the emotional subjective experience. A qualitative methodology with phenomenological-hermeneutic design was used taking as a tool conversational systems. The informants were 8 extreme athletes belonging to the same group. Extreme sports included were: Mountain climbing, rappelling, bungee jumping, and mountaineering. The results indicate that fear is a generator of freedom, it can be useful, it is rewarding and it is also a promoter of personal transformations. This research gives theoretical value to the reason for involvement in extreme sports.
\end{abstract}

Keywords: extreme sport, subjective experience, fear, transformation 


\section{Cajina-Gueudet \& Reyes-Bossio}

\section{RESUMO}

Participantes em esportes radicais geralmente têm sido estudados a partir de uma perspectiva de risco. O presente estudo busca afastar-se desse preconceito e visa estudar a experiência emocional subjetiva. Uma metodologia qualitativa com desenho fenomenológico-hermenêutico foi utilizada, utilizando sistemas conversacionais como ferramenta. Os informantes eram 8 atletas extremos pertencentes ao mesmo grupo. Esportes radicais incluem: alpinismo, rapel, bungee jumping, montanhismo e bungee jumping. Os resultados indicam que o medo é um gerador de liberdade, pode ser útil, gratificante e também promotor de transformações pessoais. Esta pesquisa dá valor teórico ao motivo do envolvimento em esportes radicais.

Palavras chave: esporte radical, experiência subjetiva, medo, transformação

\section{INTRODUCCIÓN}

Se considera que los deportes extremos nunca han sido tan populares como ahora (Pain y Pain, 2005). En los EE. UU., la participación en deportes 'alternativos' ha aumentado en 244.7 por ciento entre 1978 y 2000 (Puchan, 2004). En Perú, la Adventure Travel Trade Association (2015) refiere que es el tercer País latinoamericano con mejor y mayor crecimiento de turismo sostenible en relación al deporte de aventura. Por otro lado, PromPeru (2008) estima que el 50\% de los turistas que visitan el Perú realizan alguna actividad recreativa de aventura; asimismo estimó que los deportes alternativos son el responsable de un movimiento mayor a US\$ 1.5 millones anuales.

El deporte extremo puede ser definido como la actividad física recreativa, que lleva un riesgo de daño físico o hasta muerte (Willig, 2008). Por tal motivo, las perspectivas teóricas han procurado explicar las motivaciones de los participantes a través de marcos analíticos que se enfocan en el riesgo y enfatizan la anormalidad (Self, Henry, Findley y Reilly, 2007). Parece haber una presuposición extensamente sostenida, y conducida por la teoría, que los deportes extremos son sinónimo de riesgo y su participación es equivalente a la búsqueda de adrenalina (Delle Fave, Bassi y Massimi, 2003; Gibson y Lepp, 2008; Self et al. 2007).

Los problemas con este enfoque son que parte de la literatura revela características que no parecen encajar con esta hipótesis tradicional sobre el riesgo (Brymer, 2010; Celsi, Rose y Leigh, 1993) y además que otros aspectos parecen haber sido ignorados ocasionando que la experiencia de los participantes no sea reflejada adecuadamente (Brymer y Schweitzer, 2017; Willig, 2008). Tal cual como la investigación de Montalbetti y Chamarro (2010) evidencia en la construcción y validación de un Cuestionario de Percepción de Riesgo en Escalada: La propensión al riesgo en estos deportistas es baja.

Las publicaciones actuales en deportes extremos se pueden clasificar en dos categorías distintas, relacionadas con la personalidad; y también las vinculadas con los significados y motivaciones detrás del comportamiento de alto riesgo. La mayoría de estas investigaciones se han centrado en la búsqueda de sensaciones. Típicamente, los estudios de este tipo comparan puntuaciones de la escala de búsqueda de sensaciones (SSS) de Zuckerman obtenidas de grupos control y experimentales (Bołdak y Guszkowska, 2016; Reuter y Holder, 2016; Zheng et. al, 2015). Los resultados demuestran diferencias estadísticamente significativas entre los dos grupos, mostrando que los practicantes de deportes extremos, en efecto, demuestran una preferencia por la novedad, actividades del alto riesgo y altos niveles de excitación (Diehm y Armatas, 2004; Reuter y Holder, 2016). Por otro lado, Larkin y Griffiths (2004) analizaron la participación de cinco personas practicantes bungee-jumping y también reconocieron procesos de iniciación y mantenimiento en el deporte. 


\section{Experiencia Emocional Subjetiva en Deportistas Extremos}

Usando el Análisis Fenomenológico Interpretativo, identificaron motivos para iniciarse y quedarse implicados en el deporte. En su discusión plantean como los participantes manejan el riesgo y placer, y enfatizan que los participantes minimizan su percepción de riesgo, lo que resulta en un "sesgo optimista".

Sin embargo, en los últimos años, la experiencia subjetiva es un tema importante que se ha introducido al campo científico (Kuppens, Tuerlinckx, Russell y Barrett, 2013). No obstante, existe una preocupación de cuál es el enfoque que se le debe dar a la experiencia debido a su componente emocional. En este sentido, LeDoux (2000) indica que los investigadores de este movimiento deben de ingeniárselas para escapar de "los grilletes de la subjetividad" si el objetivo de la investigación de la emoción es prosperar. Este planteamiento evidencia el entendimiento empobrecido de la experiencia subjetiva de la emoción, la cual es un legado conductista de la psicología, que evita la fenomenología y caracteriza a los estados mentales solamente por sus causas (Halling, King y Valle, 2013). Por consiguiente, se ha pensado que conocer las causas de la emoción es un supuesto suficiente para contestar a la pregunta de cómo es la experiencia, aunque esto presupone dejar de lado cómo se siente una persona cuando tiene una experiencia. Por lo tanto, para el ámbito científico es necesario explorar como se sienten dichas experiencias y no solo sus causas (Mesquita y Boiger, 2014).

La mayoría de las teorías en relación a la emoción han sido mediante un modelo de causa-efecto o estímulo-respuesta (Forgas, 2012). Es decir que el enfoque cognitivo predomina en este ámbito. Sin embargo, según Searle (2015), es un error confundir la evidencia que tenemos sobre una materia por la materia en sí misma; el tema de la psicología es la mente humana, el comportamiento humano es evidencia y característica de la mente, pero no es la mente en sí misma.
Por lo tanto, una forma adecuada de abordar la experiencia subjetiva emocional requiere más que solo una especificación de las causas; también requiere una descripción del contenido, lo que es común en todas las experiencias de emoción, y es lo que, también, diferencia a una experiencia de otra (Feldman, Mesquita, Ocshner y Gross, 2007). En este sentido, la experiencia subjetiva se trata desde la consciencia de una emoción (Rocca, 2012). Se considera que esta consciencia de la experiencia debe ser ontológicamente subjetiva, rica en contenido, puede hacer referencia a otra cosa u otra experiencia, y está constituida por la consciencia de la emoción y no la emoción definida por procesos biológicos (Searle, 2015).

Asimismo, estos estados conscientes existen solo desde el punto de vista de la primera persona. Al ser ontológicamente subjetivos solo existen cuando se experimentan por un agente consciente y no pueden ser redefinidos independientemente del experimentador (Feldman et al., 2007).

En esencia, la experiencia subjetiva emocional es una forma de respuesta afectiva que funciona como un tipo de conocimiento sobre si los objetos, eventos o emociones son útiles o perjudiciales, gratificantes o amenazantes; o aceptados o rechazados (Oatley y Johnson-Laird, 2014).

Por ello, para entender mejor las experiencias de los participantes en deportes extremos, la presente investigación está dirigida a obtener una descripción más completa en comparación con el enfoque conductista de estímulo-respuesta y el tratamiento desde el enfoque de búsqueda de riesgo. El foco de atención de este estudio son las experiencias emocionales subjetivas. Esta investigación busca conocer las experiencias subjetivas emocionales de un grupo de practicantes de deportes extremos. Asimismo, observar los rituales y comportamientos en el ambiente que practican dicho deporte extremo. El objetivo general es describir la experiencia subjetiva emocional del grupo "los saltadores", los cuales practican dicha actividad en el Cañon de Autisha, Huarochirí. En síntesis, la presente 


\section{Cajina-Gueudet \& Reyes-Bossio}

investigación busca otorgar un valor teórico al entendimiento de la experiencia de los participantes en deportes extremos más allá de la perspectiva de riesgo y estímulo-respuesta.

\section{MATERIAL Y MÉTODOS}

\section{Diseño}

Se emplea el enfoque fenomenológicohermenéutico propuesto por González-Rey (2013) para estudiar la subjetividad llamado Epistemología Cualitativa. Para ello se utilizarán los sistemas conversacionales propuestos por el autor. En ese sentido, el diálogo es la forma en como las personas expresan las configuraciones subjetivas.

González Rey (2008) propone la categoría de sentido subjetivo como "una unidad simbólicoemocional que se organiza en la experiencia social de la persona" (p.228), en un proceso en el que se construyen y definen complejas configuraciones sobre lo experimentado.

Por otro lado, como parte del estudio de la subjetividad, la presente investigación fue hecha en la localidad de Autisha. Ya que el acceso a la vida cotidiana de los sujetos es una condición esencial para producir información acerca de cualquier aspecto en el estudio de la subjetividad (González-Rey, 1998).

Se analizó la información siguiendo la propuesta metodológica de González-Rey (2013) con la finalidad de poder identificar los trechos de información y zonas de sentido de los informantes. Cabe recalcar que, en el análisis de datos, no se hizo uso de un software como apoyo en el análisis. Debido a la cantidad de la información recolectada se pudo operacionalizar de manera manual. Asimismo, en dicha propuesta metodológica (González-Rey, 2013) se plantea que la pregunta de investigación debe responderse a través de los relatos y reflexiones de los participantes. Es así, que la conversación, como herramienta de investigación, permite registrar construcciones complejas como la experiencia emocional subjetiva, desde la particularidad de la persona que habla, en este caso, los deportistas extremos que participan en la presente investigación.

Por otro lado, este diseño podría enmarcarse dentro de los estudios observacionales como un diseño Puntual / Nomotético / Multidimensional (Anguera; Blanco Villaseñor, Hernández Mendo y Losada, 2011)

\section{Participantes}

Los informantes fueron seleccionados en base a la disponibilidad, accesibilidad y voluntad propia de participar en el estudio; asimismo todos los informantes pertenecían a la misma comunidad. Se ha considerado que los miembros pertenecen a una comunidad ya que son un grupo que en su interrelación se genera un sentido de identidad social y pertenencia, también tienen conciencia de ellos como grupo y se fortalecen como unidad social (Montero, 2005). El criterio de inclusión del estudio era que los sujetos pertenezcan al grupo de "Los Saltadores", participaron todos los miembros.

Los informantes claves estaban conformados por un grupo de 8 deportistas extremos entre 21 y 42 años, que practican puenting, trekking y rapel. Todos los informantes son solteros y su grado de instrucción va desde secundaria completa hasta superior completa. Se realizaron varios encuentros, con los informantes. Observando y conversando con cada uno a la vez.

Tabla 1

Datos generales de los informantes

\begin{tabular}{|c|c|c|c|c|}
\hline \multicolumn{3}{|c|}{$\begin{array}{l}\text { Código Edad Grado de } \\
\text { instrucción }\end{array}$} & $\begin{array}{l}\text { Estado } \\
\text { civil }\end{array}$ & Ocupación \\
\hline 001 & 27 & $\begin{array}{l}\text { Secundaria } \\
\text { Completa }\end{array}$ & Soltero & $\begin{array}{l}\text { Guía de turistas en } \\
\text { deportes de aventura }\end{array}$ \\
\hline 002 & 21 & $\begin{array}{l}\text { Superior en } \\
\text { Curso }\end{array}$ & Soltero & - \\
\hline 003 & 30 & $\begin{array}{l}\text { Superior } \\
\text { Completa }\end{array}$ & Soltero & Médico \\
\hline
\end{tabular}




\section{Experiencia Emocional Subjetiva en Deportistas Extremos}

$\begin{array}{lllrl}004 & 36 & \begin{array}{l}\text { Superior } \\ \text { Incompleta }\end{array} & \text { Soltero } & \text { Vendedor } \\ 005 & 23 & \begin{array}{l}\text { Técnico en } \\ \text { Curso }\end{array} & \text { Soltero } & \begin{array}{l}\text { Auxiliar de } \\ \text { Educación Inicial }\end{array} \\ 006 & 42 & \begin{array}{l}\text { Técnico } \\ \text { Completa }\end{array} & \text { Soltero } & \begin{array}{l}\text { Negocio Propio de } \\ \text { deportes de aventura }\end{array} \\ 007 & 28 & \begin{array}{l}\text { Secundaria } \\ \text { Completa }\end{array} & \text { Soltero } & \begin{array}{l}\text { Empleado en un } \\ \text { Hotel }\end{array} \\ 008 & 25 & \begin{array}{l}\text { Superior } \\ \text { Incompleta }\end{array} & \text { Soltero } & - \\ & & & \\ \end{array}$

En relación al contexto de la investigación, se llevó a cabo en el distrito de Huachupampa en Huarochirí. En esta localidad se encuentra el cañón de Autisha, lugar donde los informantes de la investigación practican el deporte extremo. La localidad de Autisha es popular por los deportes de aventura, el punto de atracción principal es el Puente de Autisha, y se usa para practicar puenting, deporte que consiste en atarse una cuerda elástica al torso y lanzarse desde una altura elevada. Luego los practicantes hacen rapel hacia el interior del cañón de Autisha. El descenso es de aproximadamente 170 metros. La página de Turismo Perú (2015) dice que el cañón de Autisha es el mayor atractivo turístico del distrito ya que da la ilusión de adentrarse al centro de la tierra.

La experiencia inicia cuando el grupo se encuentra para subirse al bus que los lleva hacía Huarochirí, salen de un paradero en San Borja (distrito de la ciudad de Lima) exactamente a las 5:30 a.m. El viaje dura aproximadamente 3 horas. Apenas se llega al puente, los participantes se colocan los instrumentos necesarios para lanzarse. Existen 3 formas de lanzarse: de espaldas, parado en los pasamanos del puente o "avioncito"; esta última consiste en que los miembros cargan a uno de los sujetos y lo avientan. Antes de lanzarse cada uno toma un vaso de Pisco puro, al cual le llaman "valor líquido".
Luego, se accede al cañón, para lo cual existen dos maneras: A rapel o caminando. Para acceder caminando se debe de partir del caserío más cercano que está aproximadamente a 15 minutos de caminata. La zona de la caminata es de dificultad media-alta, la zona es muy escarpada, con caminos estrechos. Para acceder al cañón a rapel se amarran unas cuerdas a rocas y se baja. La dificultad del rapel es alta. Al descender hay dos cuevas, una de ellas conduce a la que se le conoce como Cueva de Los Murciélagos, en la cual habitan estos animales. La otra es la cueva de la laguna, donde los miembros suelen descansar. Posteriormente comen trucha preparada por la tía de uno de los miembros, la cual vive en la localidad. Ahí se juntan a descansar y conversar.

Posterior a eso los participantes suelen regresarse en un bus a Lima, en el que escuchan música y bailan. Ellos le llaman "Bus Parrandero" y es con motivos de celebrar la experiencia, ahí también toman Pisco. Los participantes de la investigación realizan esta actividad aproximadamente 1 o 2 veces por semana.

\section{Instrumentos}

Se elaboró una guía de conversación. Dicha guía es un instrumento semiestructurado diseñado para orientar la dinámica conversacional hacia los objetivos de la presente investigación.

La guía de conversación fue revisada por un especialista en control de impulsos, un especialista en investigación con sistemas conversacionales y un deportista extremo; posteriormente la guía fue probada en un estudio piloto. Seguidamente a la aplicación del estudio piloto se modificó la guía para permitir que la conversación sea más fluida y evitar omitir información relevante. Una vez reelaborada fue revisada por los mismos expertos.

La conversación iniciaba con el siguiente enunciado "Cuéntame cómo es tu experiencia ¿Qué te gustaría contarme?”. La conversación fue de manera interactiva en la que se esperaba que el informante se explaye en su propia percepción de 


\section{Cajina-Gueudet \& Reyes-Bossio}

la experiencia subjetiva; en ese sentido se permitió un espacio de comunicación regulado por un diálogo organizado por áreas temáticas que giran en torno a la teoría de la experiencia subjetiva emocional.

Por otro lado, con el objetivo de abordar la subjetividad de manera más completa también se trabajó con notas de campo (en forma de notas descriptivas). En las notas de campo se registraron distancia entre los miembros, intercambio de miradas, verbalizaciones para pedir ayuda, tiempo de demora para saltar, hablar consigo mismo, gritar, expresiones faciales y demás aspectos observables.

Posteriormente, en el estudio piloto la guía fue probada por un sujeto participante en Autisha. Los resultados fueron favorables. La guía fue revisada nuevamente y validada por el juicio de expertos una vez concretado el estudio piloto. Todo ello apoyado en el carácter cualitativo de concordancia consensuada (Anguera, 1990).

\section{Procedimiento}

Primero se realizó un proceso de familiarización el cual tuvo como objetivo conocer los agentes internos y externos que participan dentro del grupo "los saltadores".

En cuanto a los aspectos éticos, los informantes firmaron un consentimiento informado en el cual se explicaron los motivos de la conversación y los objetivos de la investigación. Asimismo, se explicitó el uso de los datos de manera confidencial. Por otro lado, se notificó a los participantes que la información será recolectada mediante grabaciones de voz, registro fotográfico y notas escritas. El estudio se llevó a cabo de conformidad con la Declaración de Helsinki (Tyebkhan, 2003), que establece los principios éticos fundamentales para la investigación con seres humanos.

El estudio piloto fue aplicado a un informante que no pertenece al grupo "los saltadores", sin embargo, es un deportista extremo que visita el Cañón de Autisha.
Luego se asistió a visitas al Cañón de Autisha, durante la experiencia se observó a los miembros del grupo uno por uno, acompañándolos en su recorrido y evaluando cómo se manejaban diversas situaciones como parte de familiarización con el contexto. Posteriormente a la experiencia se realizó la conversación. Esta se centró alrededor de "cuéntame cómo es tu experiencia". De esta manera se permitía que los informantes se desplieguen en una narración profunda y completa.

Por motivos de confidencialidad se reemplazaron los nombres de los informantes por códigos.

\section{RESULTADOS}

Los resultados se construyeron siguiendo el proceso de análisis de información apoyado en la Epistemología Cualitativa de González Rey, la cual es considerada una metodología especializada el estudio de la subjetividad (Barbosa, Gandolfo y Mitjáns, 2016).

En un primer plan de trabajo se organizó la información transcrita de las conversaciones y las notas de campo en las dimensiones propuestas para la Experiencia Emocional Subjetiva (Oatley y Johnson-Laird, 2014) en una matriz de contenido. Por lo tanto, se organizaron los datos teniendo en cuenta la relación util-perjudicial, gratificante-amenazante y aceptada-rechazada.

Luego, al identificar las zonas de sentido y las citas que sustentan esa interpretación se establecen dos categorías emergentes en relación a la particularidad del caso de la experiencia subjetiva emocional en los deportistas extremos. Se reorganizaron las conversaciones en estas nuevas categorías con motivos de analizar de esta manera la experiencia subjetiva del grupo "los saltadores".

Asimismo, se pudo identificar que la emoción primaria experimentada por los informantes es el miedo. Por lo tanto, la información fue ordenada en las siguientes categorías: el miedo como generador de libertad, el miedo como sentimiento útil vs obstáculo, el miedo como sentimiento 


\section{Experiencia Emocional Subjetiva en Deportistas Extremos}

aceptado, el miedo como sentimiento gratificante y el miedo como método de transformación. Estos discursos se desencadenan del sistema de categorías de la tabla 2.

\section{Tabla 2}

Ejes de sentido y esquemas de categorías

\begin{tabular}{llll}
\hline Eje de sentido & \multicolumn{3}{l}{ Esquema de Categorías } \\
\hline BA1. Base Afectiva & BA.1.2 $\quad$ El miedo & como \\
& sentimiento útil vs. obstáculo \\
& BA.1.3 $\quad$ El miedo & como \\
& sentimiento aceptado & \\
& BA.1.4 $\quad$ El miedo & como \\
& sentimiento gratificante &
\end{tabular}

CE2.Consecuencia experiencial

CE.2.1 El miedo como generador de libertad

CE.2.2 El miedo como método de transformación

En el caso del miedo como generador de libertad, este es visto como la decisión de llevarse al límite para encontrar las cosas de las que verdaderamente uno es capaz, el participante 006 lo plantea de la siguiente manera:

"El miedo fue lo que en un principio me impidió encontrar mi propio potencial, entonces ahora sé, gracias a él también, de lo que soy capaz. El miedo, en vez de restringir mis libertades ha pasado a ser una opción. Empezó a ser mi libertad para elegir." (006)

En este mismo sentido, la decisión del miedo es considerada como generador de libertad debido a la elección de ponerse al límite, no solo para encontrar de lo que uno es capaz, sino solo por tomar la decisión en sí, más allá del logro que se pueda tener. El participante 008 se refiere a esto de la siguiente manera:

"Definitivamente es placentero... pero no es un sentimiento de logro sino este sentimiento de libertad más que cualquier otra cosa... Particularmente siento eso de que no necesito ponerme a límite en estas situaciones pero decido hacerlo de todos modos, y eso me demuestra que soy libre." (008)

Por otro lado, el miedo puede ser percibido como un obstáculo y a la vez también como un sentimiento útil. El miedo es considerado como sentimiento útil en el sentido que hace que actúes acorde a tus posibilidades y también ayuda a tener un mejor desempeño, pero a su vez te limita en algún momento.

En el caso del miedo como un obstáculo, los participantes dicen que el miedo te puede paralizar, tal como el participante 004, el cual plantea que le gustaría a veces sentirse más positivo para tener un mejor desenvolvimiento. Aun así, los participantes rescatan al miedo como un sentimiento que es predominantemente útil durante la experiencia. El participante 002 por ejemplo, indica que es el punto de claridad en cualquier momento de riesgo, ya que es el que te da todas las opciones de cómo actuar. Es así que el miedo es considerado como el encargado de mantener a salvo durante la experiencia ya que hace que uno no tome decisiones imprudentes, tal como lo plantea el participante 001:

“Alguien que te cuente que está
parado en un precipicio de 100
metros y ve abajo y no siente
nada... te está mintiendo. Eso de
no sentir nada es imposible....
Entonces ese miedo hace que te
mantengas vivo...(suspiro) ¿Por
qué? Porqué el día que dejas de
sentir miedo... ese día te vas a
arriesgar a algo que te va a matar.
Entonces, ese miedo es útil en la
montaña... El miedo con respeto,
saber que subes para bajar... no
subes para quedarte..." (001)

Asimismo, el miedo es el sentimiento que te hace reaccionar en caso haya una situación de peligro. 


\section{Cajina-Gueudet \& Reyes-Bossio}

Es decir que el miedo ayuda a controlar como desempeñarse, tal como indica el participante 008:

“Eso de que el miedo es
necesario... déjame pensarlo...
solo tengo que pensar en un
paralelo, es una combinación
completa de cosas, por ejemplo, si
alguien maneja un carro y está
lloviendo, y pierde el control y en
algún momento de ese control
pierde básicamente... toda la
mierda... Es el miedo el que hace
que tomes el control de la
situación y pongas el carro
nuevamente en una posición
segura, sabes hacer la cantidad
correcta de frenada o acelerar
para salir del giro y de repente
recuperas el control... y claro, ese
miedo te puede hacer reaccionar
un poco brusco... pero es el que
hace que todo funcione (...) Ese
miedo al peligro te mantiene con
vida." (008)

Además, los informantes refieren que no solo el miedo es útil por su cuenta si no que es un sentimiento el cual tienes que escuchar, por lo tanto, y como indica la siguiente categoría el miedo debe ser aceptado. Los informantes realizan incluso un paralelo entre el miedo con control y el miedo sin control, considerando que el miedo sin control es en el cual te encuentras superado por la adrenalina y ocasiona que tomes decisiones imprudentes. Mientras que el aceptar el miedo, incluso da la percepción de que se está sumamente calmado, tal como lo plantea el participante 007:

"El truco que creo es poder controlar los miedos y luego, si tienes el control total sobre eso, entonces no te sientes estimulado por la adrenalina, pero es por eso que te sientes relajado, es casi como llegar a un estado meditativo. Eso es controlar tu miedo, abrazarlo relajarte en él." (007)

Del mismo modo, al superar este miedo no solo tiene como resultado en los participantes un efecto durante la experiencia, sino también a superar miedos en otros momentos de la vida, tal como lo informa la participante 005:

"Mahatma Gandhi dice que el no tener miedo es el rey de todas las virtudes y... hacemos estas cosas por varias razones, pero una de las razones más poderosas para mí es superar mis miedos personales que... umm... te da tan mucho más de una visión de tu ser en su potencial $y$ en sus capacidades, una vez que abrazas el miedo, puedes lograr cualquier cosa." (005)

Asimismo, esta emoción es percibida como agradable. Es decir que el miedo no tiene la connotación negativa que se le da naturalmente en la sociedad, sino que es un sentimiento valorado positivamente. Tal como indica el participante 007:

"Hay una voz en un hombro diciendo ¿por qué estás haciendo esto? Tienes miedo, y la otra voz dice, sí, pero me gusta tener miedo. Entonces este no es un miedo malo, es un miedo que llegas a disfrutar." (007)

Por último, los informantes reportan que las experiencias emocionales traen cambios incluso desde el primer salto de puenting, incluso en otras áreas de la vida:

"También fue sin duda una tremenda sensación de logro, y un impulso a la autoestima. Podría hacer que en ese momento un cliente me gritara, y pensaría, 'causa, he ido lugares que te harían llorar'. E incluso hasta el 


\section{Experiencia Emocional Subjetiva en Deportistas Extremos}

día de hoy, siento que llevo eso conmigo." (002)

Es así que en relación al miedo como método de transformación, los participantes también reportan que el miedo es generador de calma, conectarse más con el sí mismo y otros; trayendo transformaciones permanentes, instantáneas y no esperadas. La naturaleza instantánea de transformación ha sido expresada por los participantes, como la participante 005 (una comprometida profesional médica con años trabajando) la cual fue persuadida a pesar de inicialmente pensar que involucrarse en esas actividades era una forma de tentar la muerte:

"Mi vida ha sido radicalmente alterada por esa elección para ese día sabes... como dije puedo rastrear mi cambio de ruta desde ese día porque ese día ... podría no haberme ido y con ese primer día, descubrí los cambios que yo podía lograr conmigo, ser una mejor persona." (005)

Otro participante lo plantea de esta manera, en la que el cambio no es considerado desde la primera experiencia pero si es una transformación abrupta:

"He estado meditando durante años y corriendo durante años por lo que el cambio fue lento, se podía ver el progreso lentamente, pero tuve un cambio explosivo en un corto período de tiempo, por lo que fue un catalizador para el crecimiento explosivo. Me encontré de verdad." (007)

Las transformaciones involucran cambios intelectuales, emocionales e incluso espirituales; y la experiencia es considerada como una situación que les cambió la vida. Asimismo, las transformaciones son constantes y duraderas.

Por otro lado, los informantes también describen que la experiencia altera radicalmente el sentido de sí mismo, sus capacidades como persona y el que tanto se estiman, tal como lo plantea La participante 003: "No era una persona segura cuando estaba en secundaria. Realmente me gusta quién soy como persona ahora y es porque me gusta quién soy como deportista."

Otra cuestión en la que la propia experiencia de miedo de los participantes es considerada como transformadora es en el acercamiento que tienen hacia la vida. Los informantes expresan que desempeñar la actividad enseña que no existen límites más allá de los creados mentalmente y que mediante el deporte de aventura llegas a acercarte a lo que verdaderamente eres capaz. Otra típica respuesta entre los informantes es que la experiencia enseña a ser humilde esto es debido a que existe esta percepción de cercanía a la muerte, en que el tiempo es percibido como efímero, y que todo puede terminar en cualquier momento, tal como lo dice el participante 001 al narrar la sensación de saltar al cañón de Autisha:

"Hay, creo, más que el sentimiento efimero. Todo tiene su propio tiempo, su propio espacio. El borde tiene su propia gravedad, como un gran agujero negro en el borde del universo. Nos acercamos al agujero con la mayor cautela, porque su gravedad tiene el poder de rasgar nuestros prejuicios, nuestro conocimiento seguro de la forma en que las cosas son, para dejarnos ver la forma en que las cosas pueden ser. Los agujeros negros tienen el poder de devolver nuestros sentimientos, a veces a cambio de nuestras vidas. Nunca he conocido a nadie que se haya mantenido en los flancos de una montaña, o que haya ido... brevemente, al mundo oscuro en el borde del abismo, y no volver cambiado. ¿cambiar cómo? Más humilde, tal vez, más 


\section{Cajina-Gueudet \& Reyes-Bossio}

consciente de la fragilidad de la vida" (001).

\section{DISCUSIÓN}

La perspectiva teórica tradicional sobre la participación en deportes extremos relaciona la experiencia con la toma de riesgos. Por el contrario, según los participantes en este estudio, los deportes extremos no tienen que ver con la necesidad de correr riesgos. Es decir, asumir riesgos no es la experiencia. Después de todo, el hecho de que un participante en deportes extremos no se aleje de la posibilidad de resultados indeseables no significa que esté persiguiendo riesgos. Los participantes de deportes extremos reconocen que las lesiones y la muerte son posibles y se comprometen a aprender sobre la tarea, el medio ambiente y su propia capacidad para reducir tales eventos no deseados. Los participantes entrevistados para este estudio consideran que prefieren tener el control, no consideran que están buscando imprudentemente el peligro y son conscientes de las posibles desventajas. Los participantes en deportes extremos tienen bastante claro que no buscan riesgos y que muchas actividades socialmente aceptables son inherentemente más peligrosas.

La noción de que los deportes extremos es una búsqueda de libertad ha sido planteada en algunos estudios (Allman, Mittelstaedt, Martin, y Goldenberg, 2009 y Breivik, 2010). Por ejemplo, Welser (1997) consideró la libertad como el foco principal de la experiencia en escalada, ya que tiene que ver con su capacidad de tomar elecciones, de liberarse del miedo y de tomar una propia responsabilidad sobre sus vidas.

Asimismo, en una investigación sobre los deportes extremos como generador de libertad (Brymer y McKenzie, 2017) se llega a la conclusión que quizá los participantes, al hacer actividades que "no deberían" estar haciendo, ganan libertad de lo que tenían inhibido, cayendo en cuenta de su potencial personal. En relación a eso, para Agiewich (1998), parte de la atracción de escalar es la libertad de explorar desafíos para empujar los límites personales.

En ese sentido, a diferencia de lo que dice la teoría de la desviación de la respuesta de miedo (Monasterio et al., 2016) no es que estas personas tengan menos capacidad para tener una activación del miedo. Existe el miedo, solo que este no es visto como una emoción de tipo aversiva, sino que por el contrario, un sentimiento aceptado y necesario para la experiencia, más que nada como se pudo ver anteriormente en los resultados, para mantener la vida. Por ejemplo, desde la perspectiva cuantitativa, y la elaboración de nuevos cuestionarios, también se ha propuesto que existen variables tales como "control emocional" y "sintomatología emocional" la cual reconoce el sentimiento de miedo en deportistas extremos (Puertas Oyague, Chamarro Lusar, y Font Cercós, 2005). Incluso en otras investigaciones con modelos de entrevista, los participantes plantean como el no tener miedo es visto como un riesgo tanto para la persona como para el grupo (Brymer y Schweitzer, 2017). Asimismo, que el éxito depende de balancear el estado natural de miedo para que sea útil y poder sobrellevar las respuestas que son un obstáculo (Buckley, 2018).

Debido a concepciones sobre el miedo, este es percibido como agradable, es así que las concepciones de los informantes llegan a asemejarse con experiencias cuasi-religiosas. En relación a eso Watson y Parker (2015) plantean como los deportes extremos tienen una parte mística en su desenvolvimiento, en el control del miedo lleva a un estado de relajación. Asimismo, Brymer y Schweitzer (2017) lo han llegado a comparar con la tradición budista; similar a una de las participantes de la presente investigación, la cual menciona que el control del miedo es el camino para la iluminación. Jones (2004), en una investigación de ultramaratonistas encuentra que tienen una experiencia mística por llevarse al extremo, incluso llegando a comparar la 


\section{Experiencia Emocional Subjetiva en Deportistas Extremos}

experiencia transpersonal con ciertas prácticas indígenas chamánicas.

Por otro lado, Lipscombe (1999) observó que la participación en tales actividades de riesgo proporciona oportunidades para la autorrealización, autodeterminación y humildad. En investigaciones más recientes, como la de Watson y Parker (2015), se concluye que la mente del atleta se puede entender mucho con las experiencias pico; dicha tesis establece que la experiencia en deporte extremo forma un "misticismo natural" que conlleva al bienestar del deportista. Emmons (1999) llega a una conclusión similar afirmando que eventos estresantes ayudan al crecimiento personal: mejorando la percepción de la realidad, la evaluación positiva del sí mismo, dando mayor sentido de vida, autoeficacia y comprensión de los eventos.

En relación a la autoeficacia esta se ha definido como la fuerza de la percepción de confianza o creencia de un individuo de que él o ella puede completar adecuadamente una tarea mediante la expresión de una habilidad (Maddux, 2016). Sin embargo, es interesante cómo este sentimiento de autoeficacia es independiente de la experiencia ya que en el momento de desempeñar la actividad existen en el total de los participantes alguna sensación de que las cosas pueden ir mal e incluso les gustaría tener una mejor actitud al momento que saltan o realizan el rapel. Por lo tanto, esta sensación de construcción autoeficacia no es, a diferencia de lo que otras investigaciones proponen (Baretta, Greco y Steca, 2017; García y Zubiaur González, 2019; Sánchez y Torregrosa, 2005), una característica previa al involucramiento de deportes extremos debido a no existir una percepción de que un riesgo pueda ocurrir debido al dominio propio de la actividad, sino que los participantes reportan una sensación de autoeficacia en otras áreas de su vida al involucrarse en este tipo de deporte; siendo más una experiencia transformadora.

El concepto de autoeficacia fue el único encontrado dentro de las investigaciones para resaltar las diferencias entre los deportistas extremos y los grupos de alto riesgo (Llewelyn y Sanchez, 2008; Slanger y Rudestam, 1997). Asimismo, las construcciones de autoeficacia también se llegan a encontrar en deportistas clásicos, con la diferencia de que en deportistas clásicos la autoeficacia es correlacional al tiempo involucrado en el deporte, (Capote, Díaz-Ferrer, Mata-Martín y Vila, 2017) mientras que en deportistas extremos la percepción de autoeficacia es inmediata (Shoham, Rose y Kahle, 2000).

Por otro lado, como se mencionó anteriormente, los participantes comparan sus transformaciones con tradiciones budistas, hindúes, taoístas y chamánicas. Esto es similar a la tesis de Hanna (1993) la cual refiere que la transformación resultante de la experiencia deportiva extrema puede ser similar a la sensación transpersonal o mística. Esta transformación es profunda y da una sensación de trascendencia, libertad, autoconocimiento y conciencia (Hanna, 1993).

Desde otra perspectiva, Celsi et al. (1993) consideraron que los eventos extremos son una manifestación del "ser verdadero" de una persona, lo que lleva a un cambio de filosofía de vida perdurable por la activación del "verdadero self", ajeno a las construcciones sociales. Asimismo, otras investigaciones encontradas en relación a las transformaciones en deportes extremos reportan que la experiencia es percibida como trascendental, mística y transpersonal (Brymer y Oades, 2013; Hanna, 1993 y Robinson, 2015). Robinson (1992) consideró que el crecimiento personal puede desencadenarse a través de deportes que implican peligro ya que la intensa excitación emocional y cognitiva experimentada puede ser estímulo para mayor consciencia de sí mismo, las habilidades y la sensibilidad para ser más. Asimismo Robinson (1992) reporta cambios en la comunicación auténtica producto de que el individuo retrata a su verdadero self .

Es decir que los resultados encontrados en relación a la base afectiva en deportes extremos aportan que, a diferencia de una gran parte de investigaciones en torno a la activación de miedo 


\section{Cajina-Gueudet \& Reyes-Bossio}

en deportistas extremos (Bekaroglu, y Bozo, 2017; Buckley, 2018 y Lyng, 2018) si existe el sentimiento de miedo y no una desviación de este.

Los resultados de la exploración fenomenológica de los puntos de vida de los participantes sugieren que la experiencia deportiva extrema tiene el potencial de cambiar drásticamente las formas de ser en el mundo. Experimentar un miedo intenso puede ser un evento significativo y constructivo en la vida de los participantes, que tiene implicaciones para entender el miedo como un proceso potencialmente evolutivo $y$ transformador. Para el grupo explorado, la participación en deportes extremos parece ser una forma en la cual se experimentan valores humanos fundamentales. Esta noción amplía la perspectiva para comprender y definir ampliamente los deportes extremos, no solo como un pasatiempo o 'deporte' en un sentido tradicional, sino como un estilo de vida específica de ser humano. Es decir, las formas en que los humanos se relacionan con el mundo y los medios para explorar las capacidades físicas o psicológicas $\mathrm{y}$, en última instancia, lo que significa ser humano.

Los resultados aportan al vacío teórico del porqué las personas suelen aproximarse a este tipo de actividades. Los participantes encaran estos miedos, aceptan que el control no siempre es posible, pero aun así logran manejar la situación para tomar acciones. Además, parece que al enfrentarse a esta situación la persona llega a tener un mayor conocimiento sobre sí mismo. Estos resultados en relación a la emoción del miedo se aproximan más a la psicología de la salud, mientras se alejan de la temática con la que se ha solido estudiar a los deportes de aventura. Dichos hallazgos implican redireccionar investigaciones en el futuro en ese enfoque para permitir retratar la verdadera experiencia del grupo estudiado.

Si bien se aporta información científica útil, resulta importante mencionar algunas limitaciones. Se debe de considerar que, a pesar de la nueva información encontrada en relación a deportistas extremos, se ha explorado una comunidad de deportistas específica:

Practicantes de montañismo, rapel y puenting. Por lo tanto, quizá los resultados presenten características personales de dicho grupo en lugar de la población de deportistas extremos. Se considera necesario, por lo tanto, que futuras investigaciones sigan explorando la experiencia de los deportistas extremos, en los que se puedan incluir otros deportes extremos.

Se propone también estudios que aborden la experiencia transformadora de los deportes extremos. Se sugiere que investigaciones futuras incluyan observaciones sistemáticas, tal como la observación indirecta, con la propuesta metodológica de cuantificación de elementos (Anguera, Portell, Chacón-Moscoso y Sanduvete-Chaves, 2018), con el propósito de integrar las variables cualitativas con información cuantitativa (Anguera y HernándezMendo, 2013; Anguera, Blanco Villaseñor, Hernández Mendo y Losada, 2011).Asimismo, se espera que este grupo también sea explorado desde la metodología cuantitativa, teniendo en cuenta variables como la autotrascendencia, o estado pleno (Trujillo-Torrealva y Reyes-Bossio, 2019)

\section{CONCLUSIONES}

En conclusión, los participantes rechazan tener deseos de muerte e indican que, por el contrario, la actividad deportiva extrema les hace tener mayor acercamiento hacia la vida. La experiencia emocional subjetiva del miedo hace que describan este sentimiento como gratificante, aceptado y útil. Adicionalmente también se percibe a la emoción como generadora de libertad y transformación. Parece que la experiencia deportiva extrema permite al participante romper con las barreras personales y desarrollar una comprensión de sus propios recursos y las capacidades emocionales, cognitivas, físicas y espirituales o del bienestar. Además, tal transformación a través de la experiencia también 


\section{Experiencia Emocional Subjetiva en Deportistas Extremos}

parece accionar un cambio en filosofía personal. Esta trascendencia de una comprensión previa de ser y experimentar la vida parece transformar también su comprensión y capacidades en otros aspectos de la vida, incluyendo una comprensión más sensible de su relación con los demás. En términos generales, parecería que un yo anterior es trascendido y la vida adquiere un significado nuevo y más profundo. Por lo tanto, parece que un participante sufre un tipo de renacimiento que permite transformaciones intra-personales, interpersonales e incluso cambios extra-personales.

\section{REFERENCIAS}

1. Adventure Travel Trade Association (2013). Adventure tourism development index 2015. Washington, DC.

2. Agiewich, R. (1998). High spirited: Rock climbers find great rewards in extreme challenges. Recuperado el 3 de septiembre, 2018, de: www.seattlepi.nwsource.com/getaways/0730 98/rock30.html

3. Allman, T., Mittelstaedt, R., Martin, B., \& Goldenberg, M. (2009). Exploring the motivations of BASE jumpers: Extreme sport enthusiasts. Journal of Sport \& Tourism, 14(4), 229-247. doi: https://doi.org/10.1080/1477508090345 3740

4. Anguera, M.T. (1990). Metodología observacional. En J. Arnau, M.T. Anguera y J. Gomez Benito. Metodología de la investigación en ciencias del comportamiento (pp. 125-236). Murcia: Universidad de Murcia.

5. Anguera, M. T., Portell, M., ChacónMoscoso, S., \& Sanduvete-Chaves, S. (2018). Indirect observation in everyday contexts: concepts and methodological guidelines within a mixed methods framework. Frontiers in psychology, 9, 13. doi: https://doi.org/10.3389/fpsyg.2018.00013

6. Anguera; M. T., Blanco Villaseñor, A., Hernández Mendo, A., y Losada, J. L. (2011). Diseños observacionales: ajuste y aplicación en psicología del deporte. Cuadernos de Psicología del Deporte, 11(2),63-76

7. Anguera, M.T., y Hernández-Mendo, A. (2013). La metodología observacional en el ámbito del deporte. E-balonmano.com: Revista de Ciencias del Deporte 9(3), 135160.

8. Barbosa A., Gandolfo, M. I., y Mitjáns, A . (2016). Epistemología Cualitativa de González Rey: Una forma diferente de análisis de "datos". Tecnia, 1(1), 17-32.

9. Baretta, D., Greco, A., \& Steca, P. (2017). Understanding performance in risky sport: The role of self-efficacy beliefs and sensation seeking in competitive freediving. Personality and Individual Differences, 117, 161-165. doi: https://doi.org/10.1016/j.paid.2017.06.006

10. Bekaroglu, E., \& Bozo, Ö. (2017). The Relationship Between Attachment Styles, Emotion Regulation Strategies, and HealthPromoting Behaviors: Extreme Sports Participants Versus Non-Participants. Journal of Clinical Sport Psychology, 11(2), 89-106. doi: https://doi.org/10.1123/jcsp.2016-0023

11. Breivik, G. (2010). Trends in adventure sports in a post-modern society. Sport in society, 13(2), 260-273. doi: https://doi.org/10.1080/1743043090352 2970

12. Bołdak, A., \& Guszkowska, M. (2016). Sensation Seeking as one of the Motivating Factors for Performing Skydiving. Polish Journal of Sport and Tourism, 23(2), 94-98. doi: https://doi.org/10.1515/pjst-2016-0011

13. Brymer, E. (2010) Risk taking in Extreme Sports: A phenomenological perspective. Annals of Leisure Research, 13(1/2), 218- 


\section{Cajina-Gueudet \& Reyes-Bossio}

239.

doi: https://doi.org/10.1080/11745398.2010.9686 845

14. Brymer, E., \& Mackenzie, S. H. (2017). Psychology and the extreme sport experience. In Extreme sports medicine (pp. 3-13). Springer, Cham. doi: https://doi.org/10.1007/978-3-319-28265-7_1

15. Brymer, E., \& Oades, L. G. (2009). Extreme sports: A positive transformation in courage and humility. Journal of Humanistic Psychology, 49(1), 114-126. doi: https://doi.org/10.1177/0022167808326199

16. Brymer, E., \& Schweitzer, R. (2017). Phenomenology and the extreme sport experience. Routledge. doi: https://doi.org/10.4324/9781315661643

17. Buckley, R. C. (2018). To analyse thrill, define extreme sports. Frontiers in psychology, 9, 1216. doi: https://doi.org/10.3389/fpsyg.2018.0121 6

18. Capote, J., Díaz, S., Mata, J., \& Vila, J. (2017). Self-Efficacy and Psychophysiological responses involved in sport climbing: Comparative Analysis of Two Climbing Modalities. Recuperado a partir de: https://www.researchgate.net/publication/320 596951_Self-

efficacy_and_psychophysiological_responses _involved_in_sport_climbing_comparative_a nalysis_of_two_climbing_modalities

19. Celsi, R., Rose, R., \& Leigh, T. (1993). An exploration of high-risk leisure consumption through skydiving. Journal of Consumer Research, 20, 1-23. doi: https://doi.org/10.1086/209330

20. Delle Fave, A., Bassi, M., \& Massimini, F. (2003). Quality of experience and risk perception in high-altitude climbing. Journal of Applied Sport Psychology, 15, 82-98. doi: https://doi.org/10.1080/1041320030540 2
21. Diehm, R., \& Armatas, C. (2004). Surfing: An avenue for socially acceptable risk-taking, satisfying needs for sensation seeking and experience seeking. Personality \& Individual Differences, 36(3), 663-677. doi: https://doi.org/10.1016/S01918869(03)00124-7

22. Emmons, R. (1999). The Psychology of Ultimate Concerns: Motivation and Spirituality in Personality. NY: The Guildford Press.

23. Feldman, L., Mesquita, B., Ocshner, K., \& Gross, J. (2007) The experience of Emotion. Annual Reviews Psychology, 58, 373- 403. doi:

https://doi.org/10.1146/annurev.psych.58.110 405.085709

24. Forgas, J. (2012). Affect in social thinking and behavior. Psychology Press. doi: https://doi.org/10.4324/9780203720752

25. García, M. D., \& Zubiaur González, M. (2019). Analysis of the perception of selfefficacy in paragliding pilots. Revista de Psicologia del Deporte, 28(2), 41-48.

26. Gibson, H. \& Lepp, A. (2008). Sensation seeking and tourism: Tourist role, perception of risk and destination choice. Tourism Management, 29(4), 740-750. doi: https://doi.org/10.1016/j.tourman.2007. 08.002

27. González Rey, F. (1998). Lo cualitativo y lo cuantitativo en la investigación de la psicología social. Revista cubana de Psicología, 17(1).

28. González Rey, F. (2008), Subjetividad Social, Sujeto y Representaciones Sociales, Diversitas, (4), 225-243. doi: https://doi.org/10.15332/s17949998.2008.0002.01

29. Gonzalez Rey, F. (2013). Subjetividad, cultura e investigación cualitativa en 


\section{Experiencia Emocional Subjetiva en Deportistas Extremos}

psicología: la ciencia como producción culturalmente situada. Liminales. Escritos sobre psicología y sociedad, (4), 13-36.

30. Halling S., King M. \& Valle R. (2013) An Introduction to ExistentialPhenomenological Thought in Psychology, En R. Valle \& S. Halling (Eds.), Existentialphenomenological perspectives in psychology: Exploring the breadth of human experience. Springer Science \& Business Media.

31. Hanna, F. (1993). Rigorous intuition: Consciousness, being, and the phenomenological method. The Journal of Transpersonal Psychology, 25(2), 181-197.

32. Kuppens, P., Tuerlinckx, F., Russell, J., \& Barrett, L. (2013). The relation between valence and arousal in subjective experience. Psychological Bulletin, 139(4), 917. doi: https://doi.org/10.1037/a0030811

33. Larkin, M., \& Griffiths, M. (2004). Dangerous sports and recreational drug-use: Rationalising and contextualizing risk. Journal of Community and Applied Social Psychology, 14, 215-232. doi: https://doi.org/10.1002/casp.770

34. LeDoux J. (2000) Emotion circuits in the brain. Annual Reviews of Neuroscience, 23, 155-84. doi: https://doi.org/10.1146/annurev.neuro.23.1.1 55

35. Lipscombe, N. (1999). The relevance of the peak experience to continued skydiving participation: A qualitative approach to assessing motivations. Leisure Studies, 18(4), 267-288. doi: https://doi.org/10.1080/026143699374853

36. Llewellyn, D., \& Sanchez, X. (2008). Individual differences and risk taking in rock climbing. Psychology of Sport and Exercise, 9(4), 413-426. doi: https://doi.org/10.1016/j.psychsport.2007.07. 003
37. Lyng, S. (2018). Excitement: Risk and authentic emotion. In Emotions, Everyday Life and Sociology (pp. 98-113). Routledge. doi: https://doi.org/10.4324/97813152077287

38. Maddux, J. (2016). Self-efficacy. In Interpersonal and Intrapersonal Expectancies (pp. 55-60). Routledge. doi: https://doi.org/10.4324/9781315652535-5

39. Mesquita, B., \& Boiger, M. (2014). Emotions in context: A sociodynamic model of emotions. Emotion Review, 6(4), 298-302. https://doi.org/10.1177/1754073914534480

40. Monasterio, E., Mei-Dan, O., Hackney, A., Lane, A. R., Zwir, I., Rozsa, S., \& Cloninger, C. (2016). Stress reactivity and personality in extreme sport athletes: The psychobiology of BASE jumpers. Physiology \& behavior, 167, 289-297.

doi: https://doi.org/10.1016/j.physbeh.2016.09.02 5

41. Montalbetti, T., y Chamarro, A. (2010). Construcción y validación del cuestionario de percepción de riesgo en escalada de roca. Cuadernos de Psicología del Deporte, 10(2), 43-56.

42. Montero, M. (2005). Introducción a la psicología comunitaria. Desarrollo, conceptos y procesos. Santiago del Estero, Argentina: Paidós.

43. Oatley, K., \& Johnson-Laird, P. (2014). Cognitive approaches to emotions. Trends in cognitive sciences, 18(3), 134-140. doi: https://doi.org/10.1016/j.tics.2013.12.004

44. Puertas Oyague, G., Chamarro Lusar, A., \& Font Cercós, J. (1). Propiedades psicométricas del cuestionario de habilidades psicológicas en escalada deportiva. Cuadernos De Psicología Del Deporte, 5.

45. Pain, M. T. \& Pain, M. A. (2005). Essay: Risk taking in sport. The Lancet, 366(1), 33- 34. doi: $\quad$ https://doi.org/10.1016/S01406736(05)67838-5 


\section{Cajina-Gueudet \& Reyes-Bossio}

46. PromPerú (2008) Perfil del turista de aventura. PromPerú. Recuperado a partir de: https://www.promperu.gob.pe/TurismoIN/Up loads/temp/Uploads_mercados_y_segmentos _segmentos_1005_Publicacion-Perfil-delTurista-de-Aventura.pdf

47. Puchan, H. (2004). Living 'extreme': Adventure sports, media and commercialisation. Journal of Communication Management, 9(2), 171-178. doi:

https://doi.org/10.1108/13632540510621588

48. Reuter, A. J., \& Holder, J. T. (2016). Traditional vs. Extreme Athletes: An Exploration of Personality Indicators. Ursidae: The Undergraduate Research Journal at the University of Northern Colorado, 2(3), 5.

49. Robinson, D. W. (1992). The risk-sport process: An alternative approach for humanistic physical education. Quest, 44, 88104. doi: https://doi.org/10.1080/00336297.1992.1048 4043

50. Robinson, V. (2015). Reconceptualising the mundane and the extraordinary: a lens through which to explore transformation within women's everyday footwear practices. Sociology, 49(5), 903-918. doi: https://doi.org/10.1177/0038038515591942

51. Rocca, A. (2012). Sartre: teoría fenomenológica de las emociones, existencialismo y conciencia posicional del mundo. Nómadas, 36(4), 1. doi: http://dx.doi.org/10.5209/rev_NOMA.2012.v 36.n 4.42320

52. Sánchez, X., \& Torregrossa, M. (2005). El papel de los factores psicológicos en la escalda deportiva: un análisis cualitativo. Revista de psicologia del deporte, 14(2), 0177-194.

53. Searle, J. R. (2015). Consciousness as a Problem in Philosophy and Neurobiology.
Recuperado el 28 de Octubre de 2017 de: https://scholarworks.iu.edu/dspace/hand le/2022/18937

54. Self, D., Henry E., Findley, C. \& Reilly, E. (2007) Thrill seeking: The type T personality and extreme sports. International Journal of Sport Management and Marketing 2(1-2): 175-190.

doi. https://doi.org/10.1504/IJSMM.2007.011397

55. Shoham, A., Rose, G. M., \& Kahle, L. R. (2000). Practitioners of risky sports: A quantitative examination. Journal of Business Research, 47, 237-251. doi: https://doi.org/10.1016/S01482963(98)00093-9

56. Slanger, E., \& Rudestam, K. E. (1997). Motivation and disinhibition in high risk sports: Sensation seeking and self-efficacy. Journal of Research in Personality, 31, 355374. doi: https://doi.org/10.1006/jrpe.1997.2193

57. Trujillo - Torrealva, D., \& Reyes-Bossio, M. (2019). Programa de mindfulness para la reducción de la ansiedad precompetitiva en deportistas de artes marciales. Retos, 36(36), 418-426.

58. Tyebkhan G. (2003). Declaration of Helsinki: The ethical cornerstone of human clinical research . Indian J Dermatol Venereol Leprol, 69, 245-7.

59. Watson, N. J., \& Parker, A. (2015). The Mystical and Sublime in Extreme Sports: Experiences of Psychological Well-Being or Christian Revelation?. Studies in World Christianity, 21(3), 260-281. doi: https://doi.org/10.3366/swc.2015.0127

60. Welser, H. T. (1997). Finding Life: The meanings of climbing to dedicated climbers. Masters, Miami University, Oxford, Ohio.

61. Willig, C. (2008). A phenomenological investigation of the experience of taking part in 'extreme sports'. Journal of Health 


\section{Experiencia Emocional Subjetiva en Deportistas Extremos}

Psychology, 13(5), 690-702. doi: https://doi.org/10.1177/1359105307082459

62. Zheng, Y., Tan, F., Xu, J., Chang, \&., Zhang, Y., y Shen, H. (2015). Diminished P300 to physical risk in sensation seeking. Biological psychology, 107, 44-51. doi: https://doi.org/10.1016/j.biopsycho.2015.03.

003 